\title{
The Staining of Polymers
}

R.W. Smith

2450 Clarke Drive, Lake Havasu City, AZ 86403

A fascinating feature of polymer staining is that for some pliable networks (those that contain $>\mathrm{C}=\mathrm{C}<$ ) staining not only highlights target sites, butalso provides rigidity to the polymer and enables room temperature microtomy. This means two staining protocols: 1) Bulk staining hardening of trimmed pyramids in preparation for ultramicrotomy, latexes and dispersions; and 2) Ultrathin section and cast thin film staining.

1) For bulk staining, the diffusivity of the staining media into the polymer network is critical and the media should be optimized for diffusion. It requires several days foraqueous osmium tetroxide to diffuse deeply enough into styrenic matrices (HIPS, ABS) to provide adequate staining-hardening depths but diethyl ether or tetrahydrofuran (THF) solutions of osmium tetroxide can provide adequate depths in matters of minutes [1]. Epoxy/Rubber matrices, especially crosslinked epoxies, require organic solvent media for effective staining. The use of organic solvent media must be carefully monitored to insure that the residence time of the solvent is not so long that it alters morphology. An intermediate between purely aqueous or monaqueous staining media can sometimes be best. Rubber particles in PVC do not stain well (or at all) when using aqueous media and organic media tend to overly swell or partially dissolve the PVC matrix. Thus, a suitable intermediate for $\mathrm{PVC} /$ rubber is a staining medium of $5 \% \mathrm{v} / \mathrm{v}$ THF in water.

Bulk staining can present artifact. Figure 1 is a TEM cross section of poly (styreneacrylonitrile) modified with discrete poly (butadiene) particles that was bulk stained by immersion of a trimmed pyramid in $2 \%$ aqueous osmium tetroxide for four days. The stained-hardened layer is five microns deep and presents two cautionary layers. First is the layer of rubber particle distortion near the surface $S$ where the pyramid was trimmed prior to staining. Microtoming the middle layer, G, yields good results. The deepest layer P, between stained and unstained particles, is where partially stained particles appear as donuts and could be wrongly interpreted as core-shell particles. A glance at the unstained matrix shows how effective the staining was for preserving morphology. A special case of bulk staining is the staining of polymer latexes (or dispersions) that is done by adding the staining media to a small volume of diluted latex. Here it is important to determine he proper dilution of the latex so that particles remain isolated upon drying. Generally this is in the order of fractional total solids.

2) Ultrathin sections and cast thin films can be stained using liquids or vapors in a straightforward manner but vapor staining is generally preferred due to ease of handling. Cryo sectioning, if available, can be used to circumvent bulk staining and has the advantage that there is less chance of artifact.

An awareness of what is being stained is essential. Mechanical deformation of some polymer systems may produce crazed matter and osmium can become trapped within the microfibrils of this matter and be confused with chemical staining. A different type of mechanical staining occurs for crystalline and amorphous regions that react differently to stains. This is a useful tool for differentiating between them since crystalline regions resist stains and amorphous regions "soak up" 
a telltale differential amount. A blend of two amorphous polymers, each with no stainablesites, may be differentiated based upon rates of stain perfusion - a phenomenon used to advantage by staining polymer blends at temperatures chosen so that one of them is near or at the glass transition temperature.

Negative staining can be useful other than for outlining particles sitting on flat surfaces. Figure 2 shows how protein layers normally present on surfaces of rubber latex particles delineate the particles in dipped products such as catheters. Aqueous silver nitrate accomplished this [2].

As the number and complexities of polymers has increased (blends, complex copolymers, blocks, grafts, core-shell particles, interpenetrating networks, amphilics, hydrogenated olefinics, etc.) so have the tactics for staining. Chemical alteration of the polymer to make it stainable, such as alkaline hydrolysis to enable osmium staining has been successful [3]. Most staining with ruthenium tetroxide is via vapor phase but aqueous immersion staining has been introduced [4]. Nonaqueous immersion stains are being used such as ruthenium tetroxide in carbon tetrachloride [5]; phosphotungstic acid in benzyl alcohol [6]; and osmium tetroxide in formaldehyde [7]. Preferential imbibition of styrene by one polymer of a blend and then staining the styrene with ruthenium tetroxide [8] introduces a new tactic that could be adapted for other systems. Multiple staining may be required, exemplified by a ruthenium tetroxide-uranyl acetate-lead citrate system [9].

Stains are aggressive chemicals and proper safety procedures must be followed.

\section{References}

[1] Riew and Smith, Journal of Polymer Science Part A 9 (1971) 2739

[2] Smith and Folt, Rubber Chemistry and Technology 50 (1977) 835

[3] Kanig and Neff, Journal of Colloid and Interface Science 29 (1975) 253

[4] Cao, Polymer Communications, 29 (1988) 66 and 67

[5] Nau, Journal of Material Science, 32 (1997) 5335

[6] Machedo, Journal of Polymer Science Part A 37 (1999) 1311

[7] Dutta, Rubber Chemistry and Technology, 65 (1992) 932

[8] Cudby, Journal of Natural Rubber, 12 (1997) 102

[9] Holsti-Miettia, Journal of Polymer Engineering and Science, 34 (1994) 395

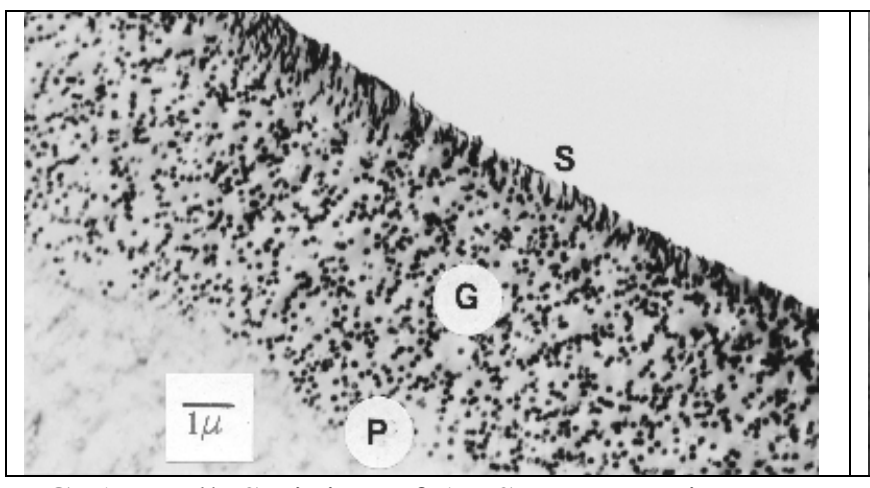

FIG. 1. Bulk Staining of ABS Type Resin Using Aqueous Osmium Tetroxide

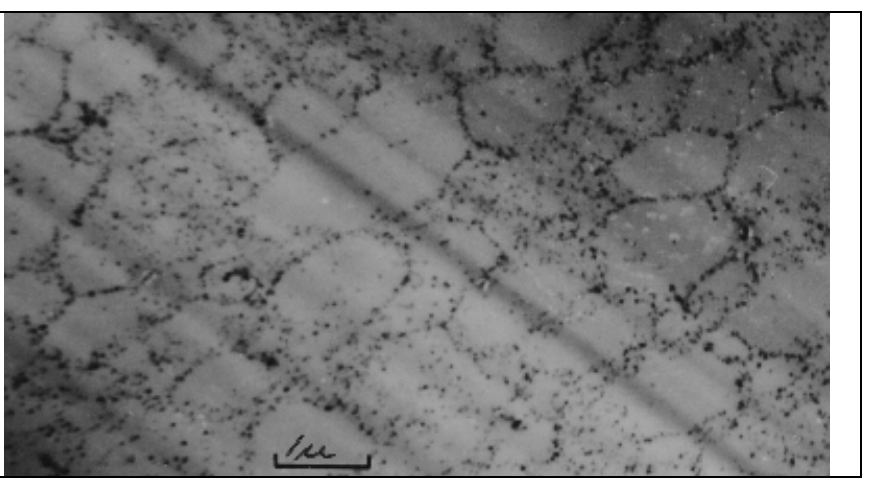

FIG. 2. Neg. Staining of NR Dipped Product Using Aqueous Silver Nitrate 